\title{
Relation Between Entrance and Exit Pupils of Telescopic Systems
}

\begin{abstract}
By Irvine C. Gardner
The definitions of the entrance and exit pupils, as commonly given, are formulated in terms of paraxial imagery only. For telescopic systems the definitions have been extended to apply to extra-axial points and to include the effects of pupil aberrations. If a telescopic system is free from distortion and an incident plane wave emerges as a plane wave free from aberrations, it is shown that exact relations between conjugate chords in the entrance and exit pupils can be derived by application of principles of the wave theory. These results are applied to give the variation in theoretical resolving power over the field of a telescopic system. Equivalent relations are derived by equating the flux of energy in the incident beam to that in the emergent beam. A relation between the illuminations of the object and image planes is derived.
\end{abstract}

Several publications ${ }^{1}$ have appeared recently in which it has been shown that there are departures from the cosine-fourth-power law governing the brightness of an image formed by an optical system that have their origin in the aberrations that exist between the entrance and exit pupils considered as conjugate areas. By conservation of energy considerations, general relations between conjugate areas in the pupils can be derived. In this discussion it is shown that for an idealized telescopic system in which the entering and emergent wave fronts are rigorously plane, it is possible, by applying the wave theory of light, to derive relations between conjugate chords in the entrance and exit pupils that indicate the presence of aberrations. The values of these aberrations are shown to be consistent with the relations between conjugate areas derivable by energy considerations to which reference has been made.

For the derivation based on the wave theory of light, let it be assumed that figure 1 represents a telescopic system that produces an image of an infinitely distant object free from all aberrations except distortion. Incident and emergent wave fronts, therefore, are assumed to be rigorously plane. An incident beam of parallel rays making

\footnotetext{
1 G. Slusareff, I. Phys. USSR 4, 537 (1941); M. Reiss, J. Opt. Soc. Am. 35, 283 (1945); I. C. Gardner, J. Rescarch NBS 39, 213 (1947) RP1824.
}

an angle, $\beta$, with the axis is shown entering the system from the left. The line $A B$ of length $\mathrm{l}$ is the trace, in the meridional plane, of the segment of the wave front that is transmitted. Similarly, $A^{\prime} B^{\prime}$, of length $\mathrm{l}^{\prime}$, is the trace of the emergent wave front corresponding to a beam of parallel rays making an angle $\beta^{\prime}$, with the axis. The value of the radial magnification, $M_{\beta}$, is defined by the equation

$$
M_{\beta}=\frac{d\left(\tan \beta^{\prime}\right)}{d(\tan \beta)}=\frac{\cos ^{2} \beta d \beta^{\prime}}{\cos ^{2} \beta^{\prime} d \beta} .
$$

If the telescopic system is free from distortion, the magnification is independent of $\beta$, and eq 1 becomes

$$
M=\tan \beta^{\prime} / \tan \beta .
$$

The dotted line, $A C$, is the trace of a second incident wave front that makes a small angle, $d \beta$, with $A B$. If $d \lambda$ represents the length $C B$,

$$
d \beta=d \lambda / l,
$$

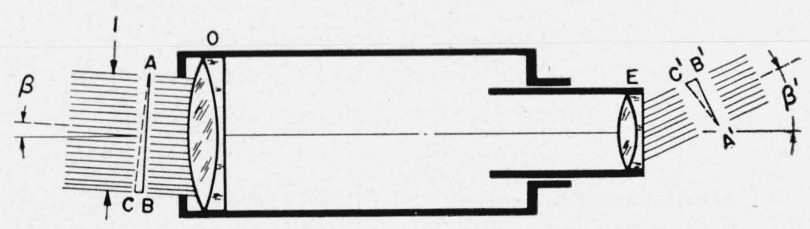

Figure 1. Diagrammatic sketch of telescopic system showing conjugate beams in the object and image spaces. 
an expression that is approximate for small values of $d \lambda$ and $d \beta$, and exact for the limit as $d \beta$ approaches zero. Similarly $A^{\prime} C^{\prime}$ represents the wave front $\mathrm{AC}$ after transmission through the telescopic system and

$$
d \beta^{\prime}=d \lambda^{\prime} / l^{\prime} .
$$

Since wave fronts $A B$ and $A C$ are conjugate to wave fronts $A^{\prime} B^{\prime}$ and $A^{\prime} C^{\prime}$ respectively, it follows that the optical paths $A \ldots A^{\prime}, B \ldots B^{\prime}$, and $C \ldots C^{\prime}$ are equal. Therefore, if the indices of refraction are the same in the object and image spaces, $B C$ and $B^{\prime} C^{\prime}$ are equal and $d \lambda_{/}^{\prime} d \lambda^{\prime}=1$. Combining eq 1,3 , and 4 ,

$$
\frac{l^{\prime}}{l}=\frac{1}{M_{\beta}} \frac{\cos ^{2} \beta}{\cos ^{2} \beta^{\prime}},
$$

which is a general relation between the diameters of right cross sections of any pair of conjugate incident and emergent beams measured in the meridional plane.

At this point it is convenient to formulate exact definitions for the entrance and exit pupils of a telescopic system applicable to object and image points not on the axis and in which the effects of aberration are not ignored. Let it be assumed that the entrance and exit pupils for paraxial rays are determined in the usual manner. These pupils determine two planes, which will be designated the pupil planes, normal to the axis of the system and conjugate in terms of first-order imagery. For an infinitely distant object at any angular distance, $\beta$, from the axis there are conjugate cylindrical beams in the object and image spaces, which have the maximum cross sections that are transmitted without obstruction by the diaphragms of the optical system. The oblique cross section of the incident beam in the entrance pupil plane is the entrance pupil corresponding to the angle $\beta$, and similarly the cross section of the emergent beam in the exit pupil plane is the exit pupil corresponding to the same object point. In general, because of the aberrations existing in the two pupil planes, the pupil areas and perimeters will vary with the angle of obliquity of the incident beams. An exception will occur in a pupil plane that contains a physical diaphragm.

Now, in accordance with these definitions, if $p$ and $p^{\prime}$ are the diameters of the entrance and exit pupils measured in the meridional plane, $p=l / \cos \beta$ and $p^{\prime}=l^{\prime} / \cos \beta^{\prime}$, respectively. Consequently, from eq 5

$$
\frac{p^{\prime}}{p}=\frac{1}{M_{\beta}} \frac{\cos ^{3} \beta}{\cos ^{3} \beta^{\prime}} .
$$

Equation 6 indicates that the entrance and exit pupils, though conjugate so far as first-order image theory is concerned, are not sharply defined but that the ratio of the meridional diameters is a function of the angles of incidence and emergence of the beams under consideration. Furthermore, since the incident and emergent wave fronts are rigorously plane, the second member of eq 6 is not only the ratio of the meridional diameters, but is also the ratio of any pair of conjugate chords, in the entrance and exit pupils respectively, parallel to a meridional plane.

Now assume that $m$ is the diameter of the entrance pupil measured in a direction at right angles to the meridional plane. Furthermore, assume that a wave front of the incident beam shown in figure 1 is rotated about an axis parallel to the meridional diameter (a rotation analogous to that illustrated in fig. 1 but with the axis of rotation turned through $90^{\circ}$ in the plane of the wave front) through the small angle $d \psi$, thereby advancing one end of the diameter through the length $d y$ from the original plane of the wave front.

then

$$
d \psi=d y / m,
$$

a relation rigorously true in the limit. As a consequence of this rotation, the azimuth of an incident ray normal to the wave front, measured as a rotation about the axis of the telescopic system, is changed by the amount $d \phi$, where

$$
d \phi=d y / m \sin \beta .
$$

For an analogous rotation in the image space, if $m^{\prime}$ is the diameter of the exit pupil, measured in a direction at right angles to the meridional plane,

$$
d \phi^{\prime}=d y^{\prime} / m^{\prime} \sin \beta^{\prime} .
$$

In a symmetrical telescopic system $d \phi$ always equals $\mathrm{d} \phi^{\prime}$.

Furthermore, as before $d y=d y^{\prime}$ if the indices of refraction of the media of the object and image spaces are the same. Combining eq 8 and 9

$$
\frac{m^{\prime}}{m}=\frac{\sin \beta}{\sin \beta^{\prime}},
$$


and a general relation between the diameters, measured in a direction normal to the meridian plane, of a pair of conjugate beams in the object and image space, respectively, has been obtained. Furthermore, by an extension similar to that previously made, the ratio of the second member of eq 10 applies not only to the two diameters but to any pair of conjugate chords, one lying in the object space and one in the image space, respectively, and normal to the meridional plane.

From. eq 6 and 10 it follows that

$$
\frac{S^{\prime}}{S}=\frac{1}{M_{\beta}} \frac{\sin \beta \cos ^{3} \beta}{\sin \beta^{\prime} \cos ^{3} \beta^{\prime}}=\frac{1}{M_{\beta}} \frac{\tan \beta \cos ^{4} \beta}{\tan \beta^{\prime} \cos ^{4} \beta^{\prime}},
$$

where $S$ and $S^{\prime}$ are the areas of the entrance and exit pupils corresponding to conjugate incident and emergent beams of rays making angles $\beta$ and $\beta^{\prime}$, respectively, with the axis.

If the telescopic system is free from distortion and is of magnification $M$, eq 11 becomes

$$
\frac{S^{\prime}}{S}=\frac{1}{M^{2}} \frac{\cos ^{4} \beta}{\cos ^{4} \beta^{\prime}},
$$

Equations 6 and 10 have interesting application to the variation of resolving power and brightness of image with angular distance from the center of the field. If a telescopic system is used for visual purposes and is so designed that the exit pupil is larger than the entrance pupil of the observer's eye, it follows that the angular limit of resolution in the image space is the same in any plane containing the axis of the eye. In the object space the corresponding angular limits of resolution in the meridional plane and in a plane at right angles to it are $\cos ^{2} \beta /\left(M \cos ^{2} \beta^{\prime}\right)$ and $\cos \beta /(M$ $\cos \beta^{\prime}$ ) times the corresponding values (which are equal) in the image space. These ratios correspond to constant values, independent of $\beta$, of the ratios $[d(\tan \beta)] /\left[d\left(\tan \beta^{\prime}\right)\right]$ and $d \phi / d \phi^{\prime}$ over the entire field.

On the other hand, if the telescopic system is so designed that the clear aperture of the objective is the limiting diaphragm of the system and the exit pupil is smaller than the eye of the observer, then the limits of angular resolution in the object space are proportional to $1 /(p \cos \beta)$ and $1 / m$, respectively, in the meridional plane and in a plane at right angles to it which contains the chief ray. The corresponding limits of resolution in the image space are proportional to $\left(M \cos ^{3} \beta^{\prime}\right) / \cos ^{3} \beta$ and $\left(M \cos \beta^{\prime}\right) / \cos \beta$, respectively. In most telescopic systems the angular field of view is so small that this variation of resolving power is not of great practical value but it is of interest as precisely defining the idealized performance of a perfect telescopic system.

It should perhaps be mentioned that a similar application of the principles of wave theory to a photographic objective is not as straight forward as the application that has just been made. If either the object or image is at a finite distance, the ratio of the tangents is not sufficient to define distortion because there is an additional contribution arising from the spherical aberration of the pupil points.

Equation 11 may also be derived by energy consideration in a manner similar to that described by Slussareff, Reiss, and others (see footnote 1). In this direction, referring to figure 2 , assume that

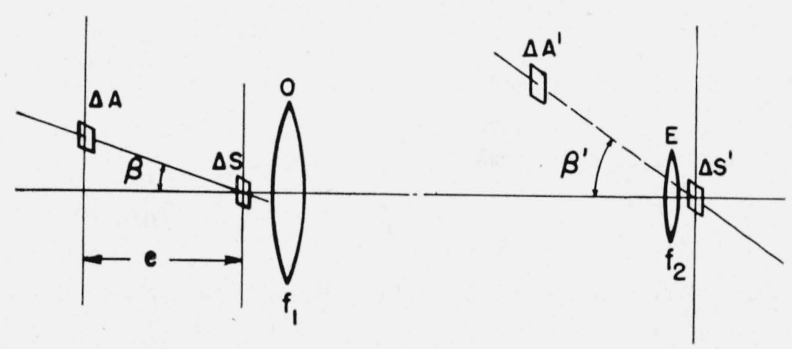

Figure 2. Diagrammatic sketch of telescopic system showing elements of area used in energy summation.

$f_{1}$ and $f_{2}$ are the objective and eyepiece parts of a telescopic system and that $\Delta A$ and $\Delta A^{\prime}$ are two conjugate elements of area in the object and image planes, respectively, of the complete system. Similarly, $\Delta S$ and $\Delta S^{\prime}$ are two conjugate elements of area in the entrance and exit pupils respectively, and $B$ and $B^{\prime}$ are the brightnesses in the directions $\beta$ and $\beta^{\prime}$ respectively, of the object and image elements. The length, $e$, is represented as finite to simplify the formulation of eq 13 , which is valid for all values of $e$. If $\Delta F$ is the flux transmitted from $\Delta A$ to $\Delta S$,

$$
\Delta F=\frac{B \Delta A \Delta S}{e^{2}} \cos ^{4} \beta^{\prime} .
$$

Similarly, for the flux transmitted from $\Delta S^{\prime}$ to $\Delta A^{\prime}$ in the image space

$$
\Delta F^{\prime}=\frac{B^{\prime} \Delta A^{\prime} \Delta S^{\prime}}{e^{\prime 2}} \cos ^{4} \beta^{\prime} .
$$


Since only the geometrical relations of the system are under consideration, it can be assumed that there are no reflection or absorption losses, in which case $\Delta F=\Delta F^{\prime}$ and $B=B^{\prime}$. Therefore

$$
\frac{\Delta S^{\prime}}{\Delta S}=\frac{\Delta A e^{\prime^{2}} \cos ^{4} \beta}{\Delta A^{\prime} e^{2} \cos ^{4} \beta^{\prime}}
$$

It is evident that

$$
\begin{gathered}
\frac{\Delta A}{\Delta A^{\prime}}=\frac{e^{2} \tan \beta \cos ^{2} \beta^{\prime} d \phi d \beta}{e^{\prime 2} \tan \beta^{\prime} \cos ^{2} \beta d \phi^{\prime} d \beta^{\prime}}, \\
\frac{d \phi}{d \phi^{\prime}}=1,
\end{gathered}
$$

and from eq 1

$$
\frac{d \beta}{d \beta^{\prime}}=\frac{1}{M_{\beta}} \frac{\cos ^{2} \beta}{\cos ^{2} \beta^{\prime}} .
$$

Substituting in eq 15 and writing $\Delta S^{\prime} / \Delta S=S^{\prime} / S$ (permissible because $\beta$ and $\beta^{\prime}$ are constant over $S$ and $S^{\prime}$, respectively) eq 11 is obtained.

It follows, therefore, that the application of the wave theory and the application of energy considerations lead to identical results so far as the relative areas of the entrance and exit pupils are concerned, but the first application gives the more detailed information in that it analyzes the areal magnification into two linear magnifications in directions mutually perpendicular.

If $E$ denotes the luminous flux emitted per unit area by $\Delta A$ and received by $\Delta S$, and $E^{\prime}$ denotes the illumination (luminous flux per unit area) received by the conjugate elementary area $\Delta A^{\prime}$ from the surface $\Delta S^{\prime}$, from eq 13 and 14

$$
\frac{E^{\prime}}{E}=\frac{\Delta S^{\prime} e^{2} \cos ^{4} \beta^{\prime}}{\Delta S e^{\prime 2} \cos ^{4} \beta} .
$$

It can be shown that

$$
\lim _{e \doteq \infty} \frac{e^{2}}{e^{\prime 2}}=\frac{f_{2}{ }^{4}}{f_{1}^{4}}=M^{4},
$$

the subscript having been dropped from $M$ because the telescopic system will be assumed free from distortion. From eq 2, 11, 19, and 20

$$
\frac{E^{\prime}}{E}=M^{2} \text {. }
$$

The ratio of illumination of image to illumination of object is independent of $\beta$, and the image is uniformly illuminated. At first eq 21 appears confusing because the illumination of the image is greater than the flux per unit area proceeding from the object, and the image is apparently larger than the object. However, it will be recalled that the linear magnification for a telescopic system is the same for all pairs of conjugate planes, including the infinite pair, and is equal to the reciprocal of the angular magnification. Also, eq 20 indicates that the two infinite planes are not equally distant but that the distance to the object plane, divided by the distance to the image plane, gives a quotient equal to the square of the magnification. As an illustration, if a ten-power telescope is assumed, linear dimensions in the image plane are one-tenth the conjugate lengths in the object plane, but the image plane is only onehundredth as far away. Hence the angular magnification is 10 , and these values accord with eq at 21 . In other words, a telescope that apparently enlarges a distant object actually produces an image that is smaller than the object, but the image is so much nearer the observer that a pair of points in the image subtend a larger angle than the conjugate pair of points in the object.

Washington, April 6, 1949. 